International Journal of Pure and Applied Mathematics

Volume 107 No. $1 \quad 2016,23-34$

ISSN: 1311-8080 (printed version); ISSN: 1314-3395 (on-line version)

url: http://www.ijpam.eu

doi: 10.12732/ijpam.v107i1.3

ijpam.eu

\title{
AN APPROXIMATE SOLUTION OF FREDHOLM INTEGRAL EQUATION OF THE SECOND KIND BY BAND-LIMITED SCALING FUNCTION
}

\author{
Gopal Priyadarshi ${ }^{1}$, B.V. Rathish Kumar ${ }^{2}$ \\ ${ }^{1}$ Department of Mathematics \& Statistics \\ Indian Institute of Technology Kanpur \\ Kanpur, 208016, INDIA
}

\begin{abstract}
Fredholm integral equation of the second kind appears naturally in different areas of mathematics, physics and engineering. In this paper we consider Fredholm integral equation of the second kind with convolution type kernel. We assume $f$ in the approximation space and prove the existence and uniqueness of approximate solution by band-limited scaling function. Since these functions are infinitely differentiable and possess decay property, methods based on these functions would be very accurate. Convergence analysis has been discussed to validate our approximate solution.
\end{abstract}

AMS Subject Classification: 41B05, 45L05, 65G99

Key Words: scaling function, orthonormal wavelet, band-limited wavelet, approximation space, Fredholm integral equation of the second kind, convolution

\section{Introduction}

Integral equations arise naturally in various fields of science and engineering [1]. These equations are encountered in a variety of applications from many fields including potential theory, mathematical physics, quantum mechanics, acoustics and fluid mechanics [2,3]. Because of its applicability in various fields, these equations have been studied extensively both at theoretical and compuational level; see $[1,2,3]$. They are closely related to differential equations. A

Received: January 16, 2016

Published: March 23, 2016

${ }^{\S}$ Correspondence address (c) 2016 Academic Publications, Ltd.

url: www.acadpubl.eu 
large class of initial and boundary value problems can be converted to Volterra or Fredholm integral equations.

In this paper we study existence and uniqueness of the approximate solution of Fredholm integral equation of the second kind with convolution type kernel which is given by

$$
u(x)=f(x)+\int_{\mathbb{R}} k(x-y) u(y) d y .
$$

Here $u(x)$ is the unknown function while $f(x)$ is a known right-hand side. The function of two variable $k(x, y)$ is called kernel.

The subject area of wavelets, developed mainly in the last two decades, has attracted people from various branches of science and technology. Due to time-frequency localization property, these functions are widely used in image processing, signal processing and data compression $[6,7]$. Haar wavelet is the simplest example of wavelet but it is not smooth. Smoother class of wavelets were constructed by Meyer in [17], Lemarie in [18], Daubechies in [8], Battle in [19] and Stromberg in [16]. We use infinitely differentiable band-limited scaling function to get the approximate solution of Fredholm integral equation of the second kind.

In the past few years many authors have studied approximate solution of Fredholm integral equation of the second kind using wavelets; see [10, 11, 12, 13]. Authors have used Haar wavelets, multiwavelets and B-spline wavelets. Haar wavelets and multiwavelets do not have sufficient regularity while B-spline wavelets does not form orthonormal basis. Regularity in basis functions and orthonormality properties, both are essential to get accurate solution of integral equations. In 2005, Shim et al. used Meyer wavelet to prove the existence of solution of Fredholm integral equation of the first kind. We provide an alternate way to find approximate solution of Fredholm integral equation of the second kind. We apply infinitely differentiable band-limited scaling function which is generated by a class of band-limited wavelets. Since these band-limited scaling functions are infinitely differentiable and possess sufficient decay property, methods based on these functions would provide accurate results for both partial differential equations and integral equations. Also convergence analysis in the setting of band-limited wavelets can be done easily comparing to other wavelets.

The content of this paper is organized as follows. In Section 2, we summarize some basics of orthonormal wavelets and multiresolution analysis. In Section 3, we consider a class of band-limited wavelets and prove a lemma. In Section 4, we prove the existence of approximate solution by band-limited scaling function generated by a class of band-limited wavelets. Convergence analysis is studied 
in Section 5. Section 6 concludes the paper and gives a brief idea of the future work.

\section{Orthonormal Wavelets and Multiresolution Analysis}

Good references for orthonormal wavelets and multiresolution analysis are [4, $5,6,7,8,17]$. Wavelets are square integrable functions generated from one scaling function by dilations and translations. These functions possess several properties such as arbitrary regularity, high order vanishing moments and an ability to represent functions at different resolutions.

Definition 1 (see [5]). An orthonormal wavelet on $\mathbb{R}$ is a function $\psi \in L^{2}(\mathbb{R})$ such that

$$
\left\{\psi_{[j, k]}(x): j, k \in \mathbb{Z}\right\},
$$

is an orthonormal basis of $L^{2}(\mathbb{R})$, where

$$
\psi_{[j, k]}(x)=2^{j / 2} \psi\left(2^{j} x-k\right), \quad j, k \in \mathbb{Z} .
$$

Definition 2 (see [5]). A multiresolution analysis (MRA) of $L^{2}(\mathbb{R})$ consists of a sequence of closed subspaces $V_{j}(j \in \mathbb{Z})$ of $L^{2}(\mathbb{R})$ satisfying

(i) $V_{j} \subset V_{j+1}$ for all $j \in \mathbb{Z}$;

(ii) $f \in V_{j} \Leftrightarrow f(2(\cdot)) \in V_{j+1} \quad$ for all $j \in \mathbb{Z}$;

(iii) $\bigcap_{j \in \mathbb{Z}} V_{j}=\{0\}$;

(iv) $\overline{\bigcup_{j \in \mathbb{Z}} V_{j}}=L^{2}(\mathbb{R})$;

(v) There exists a function $\phi \in V_{0}$ (known as scaling function) such that $\{\phi(\cdot-k): k \in \mathbb{Z}\}$ is an orthonormal basis for $V_{0}$.

Remark 3. Most of the orthonormal wavelets are constructed using multiresolution analysis theory.

Approximation space $V_{j}$ is defined as

$$
V_{j}=\overline{\operatorname{span}\left\{\phi_{[j, k]}(x) \in L^{2}(\mathbb{R}) \mid k \in \mathbb{Z}\right\}},
$$

where

$$
\phi_{[j, k]}(x)=2^{j / 2} \phi\left(2^{j} x-k\right), \quad j, k \in \mathbb{Z} .
$$


In a similar way, wavelet space $W_{j}$ is defined as

$$
W_{j}=\overline{\operatorname{span}\left\{\psi_{[j, k]}(x) \in L^{2}(\mathbb{R}) \mid k \in \mathbb{Z}\right\}},
$$

where

$$
\psi_{[j, k]}(x)=2^{j / 2} \psi\left(2^{j} x-k\right), \quad j, k \in \mathbb{Z} .
$$

Some properties of scaling function in the frequency domain:

(i) $\hat{\phi}(\xi)$ is continuous and $\hat{\phi}(0) \neq 0$.

(ii) $\sum_{k \in \mathbb{Z}}|\hat{\phi}(\xi+2 k \pi)|^{2}=1, \quad$ for a.e. $\xi \in \mathbb{R}$.

The orthogonal projection $P_{j}: L^{2}(\mathbb{R}) \rightarrow V_{j}$ is defined as

$$
P_{j} f=\sum_{k \in \mathbb{Z}}\left\langle f, \phi_{[j, k]}\right\rangle \phi_{[j, k]},
$$

where $\langle$.$\rangle denotes the standard inner product in L^{2}(\mathbb{R})$.

In a similar way, a function can be represented in wavelet space. The orthogonal projection $Q_{j}: L^{2}(\mathbb{R}) \rightarrow W_{j}$ is defined as

$$
Q_{j} f=\sum_{k \in \mathbb{Z}}\left\langle f, \psi_{[j, k]}\right\rangle \psi_{[j, k]} .
$$

Since the family $\left\{\psi_{[j, k]}(x): j, k \in \mathbb{Z}\right\}$ forms an orthonormal basis for $L^{2}(\mathbb{R})$, every function $f \in L^{2}(\mathbb{R})$ admits the $L^{2}(\mathbb{R})$ convergent wavelet expansion

$$
f=\sum_{j \in \mathbb{Z}} \sum_{k \in \mathbb{Z}}\left\langle f, \psi_{[j, k]}\right\rangle \psi_{[j, k]} .
$$

Definition 4 (see [5]). A scaling function $\phi \in L^{2}(\mathbb{R})$ is said to be bandlimited scaling function if support of $\hat{\phi}$ is contained in a finite interval.

These functions are used to get the approximate solution of Fredholm integral equation of the second kind. 


\section{A Class of Band-Limited Wavelets, see [5], [9]}

In this section, we consider all orthonormal wavelets $\psi$ with $\operatorname{supp}(\hat{\psi})$ contained in $\left[\frac{-8 \pi}{3}, \frac{-2 \pi}{3}\right] \cup\left[\frac{2 \pi}{3}, \frac{8 \pi}{3}\right]$. All these wavelets are characterized by some results which we present below. Then we prove a lemma that provide us the relationship between scaling function and wavelet in the frequency domain.

Definition 5 (see [5]). An orthonormal wavelet $\psi \in L^{2}(\mathbb{R})$ is said to be band-limited wavelet if support of $\hat{\psi}$ is contained in a finite interval.

Example 6. A function $\psi \in L^{2}(\mathbb{R})$ such that its Fourier transform is given by

$$
\hat{\psi}(\xi)= \begin{cases}1, & \text { if } \xi \in[-2 \pi,-\pi] \cup[\pi, 2 \pi], \\ 0, & \text { elsewhere }\end{cases}
$$

then $\psi$ is said to be Shannon wavelet.

The following two theorems completely characterize all orthonormal wavelets $\psi$ for which $\hat{\psi}$ has support contained in $\left[\frac{-8 \pi}{3}, \frac{-2 \pi}{3}\right] \cup\left[\frac{2 \pi}{3}, \frac{8 \pi}{3}\right]$.

Theorem 7 (see [5]). Suppose $\psi \in L^{2}(\mathbb{R})$ and $b=|\hat{\psi}|$ has support contained in $\left[\frac{-8 \pi}{3}, \frac{-2 \pi}{3}\right] \cup\left[\frac{2 \pi}{3}, \frac{8 \pi}{3}\right]$. Then $\psi$ is an orthonormal wavelet if and only if:

(i) $b^{2}(\xi)+b^{2}\left(\frac{\xi}{2}\right)=1 \quad$ for a.e. $\xi \in\left[\frac{4 \pi}{3}, \frac{8 \pi}{3}\right]$,

(ii) $b^{2}(\xi)+b^{2}(\xi+2 \pi)=1 \quad$ for a.e. $\xi \in\left[\frac{-4 \pi}{3}, \frac{-2 \pi}{3}\right]$,

(iii) $b(\xi)=b\left(\frac{\xi}{2}+2 \pi\right) \quad$ for a.e. $\xi \in\left[\frac{-8 \pi}{3}, \frac{-4 \pi}{3}\right]$,

(iv) $\hat{\psi}(\xi)=e^{i \alpha(\xi)} b(\xi) \quad$ where $\alpha$ satisfies

$\alpha(\xi)+\alpha(2(\xi-2 \pi))-\alpha(2 \xi)-\alpha(\xi-2 \pi)=(2 m(\xi)+1) \pi$,

for some $m(\xi) \in \mathbb{Z}$, for a.e. $\xi \in\left[\frac{2 \pi}{3}, \frac{4 \pi}{3}\right] \cap \operatorname{supp}(b) \cap\left(\frac{1}{2} \operatorname{supp}(b)\right)$.

Theorem 8 (see [5]). Let $\psi$ be an orthonormal wavelet such that $\hat{\psi}$ is supported in $\left[\frac{-8 \pi}{3}, \frac{8 \pi}{3}\right]$; then $\hat{\psi}(\xi)=0$ for a.e. $\xi \in\left[\frac{-2 \pi}{3}, \frac{2 \pi}{3}\right]$.

Remark 9. Conditions (i), (ii) and (iii) of theorem 7 imply that $b(\xi)$ is completely determined by its values on the interval $\left[\frac{2 \pi}{3}, \frac{4 \pi}{3}\right]$.

Remark 10. If $\operatorname{supp}(b) \cap\left(\frac{1}{2} \operatorname{supp}(b)\right)$ has an empty interior on $\left[\frac{2 \pi}{3}, \frac{4 \pi}{3}\right]$, we can choose $\alpha(\xi)$ to be any measurable function; in particular $\alpha(\xi)=0$. 
Now we give some examples which belong to our class of band-limited wavelets i.e. $\psi$ is an orthonormal wavelet, support of $\hat{\psi}$ is contained in $\left[\frac{-8 \pi}{3}, \frac{-2 \pi}{3}\right] \cup$ $\left[\frac{2 \pi}{3}, \frac{8 \pi}{3}\right]$ and characterized by theorem 7 and 8 .

Example 11. We take $b(\xi)=\chi_{\left[\frac{2 \pi}{3}, \pi\right]}(\xi)$ on $\left[\frac{2 \pi}{3}, \frac{4 \pi}{3}\right]$. Conditions (i), (ii) and (iii) of theorem 7 give us

$$
b(\xi)=\chi_{\left[\frac{-8 \pi}{3},-2 \pi\right] \cup\left[-\pi, \frac{-2 \pi}{3}\right] \cup\left[\frac{2 \pi}{3}, \pi\right] \cup\left[2 \pi, \frac{8 \pi}{3}\right]}(\xi) \quad \text { on } \mathbb{R} .
$$

Since $\left[\frac{2 \pi}{3}, \frac{4 \pi}{3}\right] \cap \operatorname{supp}(b) \cap\left(\frac{1}{2} \operatorname{supp}(b)\right)=\left\{\frac{2 \pi}{3}\right\}$, from remark 10, we can choose $\alpha(\xi)$ to be any measurable function; in particular we can take $\alpha(\xi)=\frac{\xi}{2}$.

Then

$$
\hat{\psi}(\xi)=e^{i \frac{\xi}{2}} b(\xi)
$$

make $\psi$ an orthonormal wavelet.

Remark 12. In a similar way, we can construct many wavelets. For example, Shannon wavelet and Lemarie - Meyer wavelet can be constructed in the same way.

Lemma 13. For the wavelets characterized by Theorem 7 and 8, we have

$$
|\hat{\phi}(\xi)|^{2}= \begin{cases}1, & \text { if }|\xi| \leq \frac{2 \pi}{3} \\ b^{2}(2 \xi), & \text { if } \frac{2 \pi}{3}<|\xi| \leq \frac{4 \pi}{3} \\ 0, & \text { if }|\xi|>\frac{4 \pi}{3}\end{cases}
$$

Proof. We know that

$$
|\hat{\phi}(\xi)|^{2}=\sum_{j=1}^{\infty}\left|\hat{\psi}\left(2^{j} \xi\right)\right|^{2}, \text { for a.e. } \xi \in \mathbb{R} \text {, see [5]. }
$$

Case I. $|\xi| \leq \frac{2 \pi}{3}$.

We fix a $\xi$ such that $0<\xi<\frac{2 \pi}{3}$. Now consider a sequence $\left\{2^{j} \xi\right\}_{j=1}^{\infty}$. It is clear that there exists a unique $n \in \mathbb{N}$ s.t.

$$
2^{n-1} \xi<\frac{2 \pi}{3} \text { and } 2^{n} \xi>\frac{2 \pi}{3} .
$$

(2) implies $2^{n+1} \xi>\frac{4 \pi}{3}$. Similarly, $2^{n+2} \xi>\frac{8 \pi}{3}$. Since support of $b(\xi)$ is contained in $\left[\frac{-8 \pi}{3}, \frac{-2 \pi}{3}\right] \cup\left[\frac{2 \pi}{3}, \frac{8 \pi}{3}\right], b\left(2^{j} \xi\right)=0$ for $j \geq n+2$. It follows that

$$
|\hat{\phi}(\xi)|^{2}=b^{2}(2 \xi)+b^{2}(4 \xi)+\ldots .+b^{2}\left(2^{n+1} \xi\right) .
$$


From theorem $8, b\left(2^{k} \xi\right)=0, k=1,2, \ldots ., n-1$, for $0<2^{n-1} \xi<\frac{2 \pi}{3}$. We get

$$
|\hat{\phi}(\xi)|^{2}=b^{2}\left(2^{n} \xi\right)+b^{2}\left(2^{n+1} \xi\right)
$$

Let $x=2^{n+1} \xi$. If $x \in\left[\frac{4 \pi}{3}, \frac{8 \pi}{3}\right]$ a.e. then condition (i) of theorem 7 implies $b^{2}(x)+b^{2}\left(\frac{x}{2}\right)=1$ i.e. $|\hat{\phi}(\xi)|^{2}=1$. We already have $2^{n+1} \xi>\frac{4 \pi}{3}$ from (2). So it is enough to show that $2^{n+1} \xi \leq \frac{8 \pi}{3}$.

Suppose $2^{n+1} \xi>\frac{8 \pi}{3}$. From (2) $2^{n+1} \xi<\frac{8 \pi}{3}$, which is a contradiction.

Now fix a $\xi$ such that $\frac{-2 \pi}{3}<\xi<0$. It is clear that there exists a unique $n \in \mathbb{N}$ s.t.

$$
2^{n-1} \xi>\frac{-2 \pi}{3} \text { and } 2^{n} \xi<\frac{-2 \pi}{3} .
$$

(3) implies $2^{n+1} \xi<\frac{-4 \pi}{3}$. Similarly, $2^{n+2} \xi<\frac{-8 \pi}{3}$. Since support of $b(\xi)$ is contained in $\left[\frac{-8 \pi}{3}, \frac{-2 \pi}{3}\right] \cup\left[\frac{2 \pi}{3}, \frac{8 \pi}{3}\right], b\left(2^{j} \xi\right)=0$ for $j \geq n+2$. We get

$$
|\hat{\phi}(\xi)|^{2}=b^{2}(2 \xi)+b^{2}(4 \xi)+\ldots .+b^{2}\left(2^{n+1} \xi\right) .
$$

From theorem $8, b\left(2^{k} \xi\right)=0, k=1,2, \ldots, n-1$, for $\frac{-2 \pi}{3}<2^{n-1} \xi<0$. It follows that

$$
|\hat{\phi}(\xi)|^{2}=b^{2}\left(2^{n} \xi\right)+b^{2}\left(2^{n+1} \xi\right)
$$

Let $x=2^{n} \xi$ then $|\hat{\phi}(\xi)|^{2}=b^{2}(x)+b^{2}(2 x)$. From $(3), \frac{-4 \pi}{3}<x<\frac{-2 \pi}{3}$. Similarly, $\frac{-8 \pi}{3}<2 x<\frac{-4 \pi}{3}$.

From condition (iii) of theorem 7 we have $b(2 x)=b(x+2 \pi)$. From condition (ii) of theorem $7, b^{2}(x)+b^{2}(2 x)=1$, i.e. $|\hat{\phi}(\xi)|^{2}=1$. When $\xi=\frac{2 \pi}{3}$, condition (i) of theorem 7 gives us $|\hat{\phi}(\xi)|^{2}=1$.

So we have $|\hat{\phi}(\xi)|^{2}=1$.

Case II. $\frac{2 \pi}{3}<|\xi| \leq \frac{4 \pi}{3}$.

In this case, $|\hat{\phi}(\xi)|^{2}=b^{2}(2 \xi)$. All other terms $b^{2}(4 \xi), b^{2}(8 \xi), \ldots$ vanish as the support of $b(\xi)$ is contained in $\left[\frac{-8 \pi}{3}, \frac{-2 \pi}{3}\right] \cup\left[\frac{2 \pi}{3}, \frac{8 \pi}{3}\right]$.

Case III. $|\xi|>\frac{4 \pi}{3}$. 
In this case, $|\hat{\phi}(\xi)|^{2}=0$ as the support of $b(\xi)$ is contained in $\left[\frac{-8 \pi}{3}, \frac{-2 \pi}{3}\right] \cup$ $\left[\frac{2 \pi}{3}, \frac{8 \pi}{3}\right]$.

The class of band-limited wavelets which is characterized by theorem 7 and 8 can be generated by a multiresolution analysis. We choose $\hat{\phi}(\xi)=e^{i \alpha(2 \xi)}|\hat{\phi}(\xi)|$ to obtain $\psi$ from a multiresolution analysis. For appropriate choice of $\alpha$ we have $\hat{\phi}(\xi)=|\hat{\phi}(\xi)|$. For those $\alpha$ we have

$$
\hat{\phi}(\xi)= \begin{cases}1, & \text { if }|\xi| \leq \frac{2 \pi}{3} \\ b(2 \xi), & \text { if } \frac{2 \pi}{3}<|\xi| \leq \frac{4 \pi}{3} \\ 0, & \text { if }|\xi|>\frac{4 \pi}{3}\end{cases}
$$

\section{Existence of Approximate Solution by a Band-Limited Scaling Function}

Theorem 14. Let $k \in L^{1}(\mathbb{R})$ is continuous and $f \in V_{j}$. If $\hat{k}(\xi) \neq 1$ in $\left[-2^{j+2} \frac{\pi}{3}, 2^{j+2} \frac{\pi}{3}\right]$, then integral equation (1) has unique solution in $V_{j+1}$.

Proof. From (1), we have

$$
u(x)=f(x)+(k * u)(x) .
$$

Taking Fourier transform on both sides, we get

$$
\begin{array}{r}
\hat{u}(\xi)=\hat{f}(\xi)+\hat{k}(\xi) \hat{u}(\xi), \\
\hat{u}(\xi)=\frac{\hat{f}(\xi)}{1-\hat{k}(\xi)} .
\end{array}
$$

Since $f \in V_{j}$

$$
f(x)=\sum_{k \in \mathbb{Z}} d_{k} \phi_{[j, k]}(x),
$$

where

$$
d_{k}=\int_{\mathbb{R}} f(x) \phi_{[j, k]}(x) d x .
$$


Taking Fourier transform of $f(x)$, we get

$$
\begin{aligned}
\hat{f}(\xi) & =\int_{\mathbb{R}} f(x) e^{-i \xi x} d x \\
& =\int_{\mathbb{R}} \sum_{k \in \mathbb{Z}} d_{k} \phi_{[j, k]}(x) e^{-i \xi x} d x \\
& =\int_{\mathbb{R}} \sum_{k \in \mathbb{Z}} d_{k} 2^{j / 2} \phi\left(2^{j} x-k\right) e^{-i \xi x} d x \\
& =2^{j / 2} \sum_{k \in \mathbb{Z}} d_{k} \int_{\mathbb{R}} \phi\left(2^{j} x-k\right) e^{-i \xi x} d x, \\
& =2^{-j / 2} \sum_{k \in \mathbb{Z}} d_{k} \int_{\mathbb{R}} \phi(y) e^{-i \xi\left(2^{-j}(y+k)\right)} d y, \\
& =2^{-j / 2} \sum_{k \in \mathbb{Z}} d_{k} e^{-i 2^{-j} \xi k} \int_{\mathbb{R}} \phi(y) e^{-i 2^{-j} \xi y} d y, \\
& =\tilde{d}_{k}(\xi) \hat{\phi}\left(2^{-j} \xi\right), \quad \text { where } \tilde{d}_{k}(\xi)=2^{-j / 2} \sum_{k \in \mathbb{Z}} d_{k} e^{-i 2^{-j} \xi k} .
\end{aligned}
$$

Notice that $\tilde{d}_{k}(\xi)$ is $2^{j+1} \pi$ periodic. Since $\hat{\phi}\left(2^{-j} \xi\right)$ has support in $\left[-2^{j+2} \frac{\pi}{3}, 2^{j+2} \frac{\pi}{3}\right]$, we have

$$
\hat{u}(\xi)= \begin{cases}\frac{\tilde{d_{k}}(\xi) \hat{\phi}\left(2^{-j} \xi\right)}{1-\hat{k}(\xi)}, & \text { if }-2^{j+2} \frac{\pi}{3} \leq \xi \leq 2^{j+2} \frac{\pi}{3} \\ 0, & \text { otherwise. }\end{cases}
$$

Extend $\frac{\hat{\phi}\left(2^{-j} \xi\right)}{1-\hat{k}(\xi)}$ periodically $2^{j+2} \pi$ and the extended function is denoted by $h(\xi)$ where

$$
h(\xi)=\sum_{l \in \mathbb{Z}} \frac{\hat{\phi}\left(2^{-j}\left(\xi+2^{j+2} l \pi\right)\right)}{1-\hat{k}\left(\xi+2^{j+2} l \pi\right)} .
$$

We note that $h(\xi)=\frac{\hat{\phi}\left(2^{-j} \xi\right)}{1-\hat{k}(\xi)}$ on $\left[-2^{j+2} \frac{\pi}{3}, 2^{j+2} \frac{\pi}{3}\right]$. Since $\hat{\phi}(\xi)=1$ on

$$
\left[\frac{-2 \pi}{3}, \frac{2 \pi}{3}\right], \hat{\phi}\left(2^{-j} \xi\right)=1
$$

on $\left[-2^{j+1} \frac{\pi}{3}, 2^{j+1} \frac{\pi}{3}\right]$ and $\hat{\phi}\left(2^{-j-1} \xi\right)=1$ on $\left[-2^{j+2} \frac{\pi}{3}, 2^{j+2} \frac{\pi}{3}\right]$.

We have

$$
\widehat{u}(\xi)=\tilde{d}_{k}(\xi) \frac{\hat{\phi}\left(2^{-j} \xi\right)}{1-\hat{k}(\xi)}
$$




$$
\begin{aligned}
& =\tilde{d}_{k}(\xi) \frac{\hat{\phi}\left(2^{-j} \xi\right)}{1-\hat{k}(\xi)} \hat{\phi}\left(2^{-j-1} \xi\right), \\
& =\tilde{d}_{k}(\xi) h(\xi) \hat{\phi}\left(2^{-j-1} \xi\right), \\
& =\tilde{n_{k}}(\xi) \hat{\phi}\left(2^{-j-1} \xi\right),
\end{aligned}
$$

where $\tilde{n_{k}}(\xi)=\tilde{d}_{k}(\xi) h(\xi)$ is a $2^{j+2} \pi$ periodic function since $\tilde{d}_{k}(\xi)$ is a $2^{j+1} \pi$ periodic function and $h(\xi)$ is a $2^{j+2} \pi$ periodic function. So we can conclude that $u \in V_{j+1}$. Since kernel is continuous, the solution is unique.

\section{Convergence Analysis}

Theorem 15 (see [14]). Let $f \in\left(L^{2} \cap C^{k}\right)(\mathbb{R}), k>0, \phi$ and $\psi$ be scaling function and wavelet respectively. Assume that $\phi, \psi \in C^{\beta}$ for some $0<\beta<k$. Also, assume that $\phi, \psi$ satisfy the following decay condition:

$$
|\phi(x)| \leq c(1+|x|)^{-1-\epsilon}, \quad|\psi(x)| \leq c(1+|x|)^{-1-\epsilon}
$$

for some constants $c, \epsilon>0$. For $J>0$, define the projection operator $P_{J}$ by

$$
P_{J}(f)=\sum_{k \in \mathbb{Z}}\left\langle f, \phi_{0, k}\right\rangle \phi_{0, k}+\sum_{j=0}^{J-1} \sum_{k \in \mathbb{Z}}\left\langle f, \psi_{j, k}\right\rangle \psi_{j, k}
$$

Then $P_{J}(f)$ converges to $f$ in the $\|.\|_{\infty}$ norm. Moreover, $\left\|P_{J}(f)-f\right\|_{\infty} \leq c 2^{-J k}$ for some constant $c$ depending only on $f$.

Remark 16. We note that theorem 15 is written in our paper's convention.

Remark 17. If $f \in L^{2}(\mathbb{R})$, then the wavelet expansion (4) converges to $f$ in $L^{2}$ - norm; see theorem 15 and [15].

Remark 18. We note that $f_{J} \in V_{J}$ implies $f_{J} \in V_{0} \oplus \oplus_{j=0}^{J-1} W_{j}$. It follows that

$$
P_{J}(f)=\sum_{k \in \mathbb{Z}}\left\langle f, \phi_{0, k}\right\rangle \phi_{0, k}+\sum_{j=0}^{J-1} \sum_{k \in \mathbb{Z}}\left\langle f, \psi_{j, k}\right\rangle \psi_{j, k} .
$$

Theorem 19. Let $u \in L^{2}(\mathbb{R})$ be the exact solution and $u_{J+1} \in V_{J+1}$ be the approximate solution. We assume that $\left|(1-\hat{k}(\xi))^{-1}\right| \leq M$, for all $\xi$. Then $u_{J+1}$ converges to $u$ in $L^{2}-$ norm.

Proof. Since $u$ is the exact solution, it satisfies

$$
u=f+k * u .
$$


Similarly, the approximate solution $u_{J+1}$ satifies

$$
u_{J+1}=f_{J}+k * u_{J+1} \text {. }
$$

From (5) and (6), we have

$$
u-u_{J+1}=\left(f-f_{J}\right)+k *\left(u-u_{J+1}\right) .
$$

Taking Fourier transform on both sides, we get

$$
\left(u-u_{J+1}\right)^{\wedge}=\left(f-f_{J}\right)^{\wedge}+\hat{k}\left(u-u_{J+1}\right)^{\wedge},
$$

It follows that

$$
\left(u-u_{J+1}\right)^{\wedge}=\frac{\left(f-f_{J}\right)^{\wedge}}{1-\hat{k}} .
$$

This implies

$$
\left\|\left(u-u_{J+1}\right)^{\wedge}\right\|_{L^{2}}=\left\|\frac{\left(f-f_{J}\right)^{\wedge}}{1-\hat{k}}\right\|_{L^{2}} .
$$

(10), Plancherel theorem [5] and assumption of the theorem imply that

$$
\left\|\left(u-u_{J+1}\right)\right\|_{L^{2}} \leq M\left\|\left(f-f_{J}\right)\right\|_{L^{2}} .
$$

From remark 17, $f_{J} \rightarrow f$ in $L^{2}$-norm as $J \rightarrow \infty$. So we can conclude that $u_{J+1} \rightarrow u$ in $L^{2}$ - norm.

\section{Conclusion and Future Work}

We have established the existence and uniqueness of approximate solution of Fredholm integral equation of the second kind with convolution type kernel using band-limited scaling function. Convergence analysis has been discussed to validate our method. In future, we will use these scaling functions to get the local solution of Fredholm integral equations. Since these band-limited scaling functions are infinitely differentiable and possess sufficient decay property, these functions would be very effective for the numerical solution of integral equations.

\section{Acknowledgments}

The first author would like to thank University Grants Commission for providing Ph.D. scholarship. Authors are grateful to the anonymous reviewers for their expert suggestions. 


\section{References}

[1] A. M. Wazwaz, Linear and nonlinear integral equations: Methods and Applications, Springer, New York (2011).

[2] K. E. Atkinson, The numerical solution of integral equations of the second kind, First ed. Cambridge University Press, Cambridge (1997).

[3] K. E. Atkinson, A survey of numerical methods for Fredholm integral equations of the second kind, SIAM, Philadelphia, (1976).

[4] David K. Ruch, Patrick J. Van fleet, Wavelet theory: an elementary approach with applications, First ed. Wiley, New York (2009).

[5] E. Hernandez, G. Weiss, A first course on wavelets, First ed., CRC Press, Florida (1996).

[6] I. Daubechies, Ten lectures on wavelets, CBMS-NSF Regional Conference series in Applied Mathematics, 61, Second ed. SIAM, Philadelphia (1992).

[7] G. Strang, T. Nguyen, Wavelets and filter banks, First ed., Wellesley-Cambridge Press, Wellesley (1996).

[8] I. Daubechies, Orthonormal basis of compactly supported wavelets, Comm. Pure Appl. Math. 41 (1988), 909-996.

[9] H. T. Shim, C. H. Park, An approximate solution of an integral equation by wavelets, J. Appl. Math. \& computing, 17 (2005), 709-717.

[10] E. Babolian, A. Shahsavaran, Numerical solution of nonlinear Fredholm integral equations of the second kind using Haar wavelets, Journal of Computational and Applied Mathematics, 225 (2009), 87-95.

[11] C. A. Micchelli, Y. Xu, Y. Zhao, Wavelet Galerkin methods for second-kind integral equations, Journal of Computational and Applied Mathematics, 86 (1997), 251-270.

[12] K. Maleknejad and M. N. Sahlan, The method of moments for solution of second kind Fredholm integral equations based on B-spline wavelets, International Journal of Computer Mathematics, 87 (2010), 1602-1616.

[13] Y. Mahmoudi, Wavelet Galerkin method for numerical solution of nonlinear integral equation, Applied Mathematics and Computation, 167 (2005), 1119-1129.

[14] A. Karoui, Wavelets: Properties and approximate solution of a second kind integral equation, Computers and Mathematics with Applications, 46 (2003), 263-277.

[15] A. Cohen, I. Daubechies, J. C. Feauveau, Biorthogonal bases of compactly supported wavelets, Comm. Pure Appl. Math. 45 (1992) 485-560.

[16] J.O. Stromberg, A modified Franklin system and higher order spline systems on $\mathbb{R}^{n}$ as unconditional bases for Hardy spaces, Conference in Honor of A. Zygmund, Vol. II, W. Beckner et al., eds., Wadsworth Math. Series, Belmont, California, (1982), 475-493.

[17] Y. Meyer, Ondelettes et operateurs, Hermann, Paris, (1990).

[18] P.G. Lemarie, Ondelettes a localisation exponentielle, J. Math. Pures et Appl. 67 (1988), 227-236.

[19] G. Battle, A block spin construction of ondelettes. Part I: Lemarid functions, Comm. Math. Phys. 110 (1997), 601-615. 